\title{
Adolescent Chronic Unpredictable Stress Exposure Is a Sensitive Window for Long-Term Changes in Adult Behavior in Mice
}

\author{
Nicole L Yohn' and Julie A Blendy*,I \\ 'Department of Systems Pharmacology and Translational Therapeutics, Perelman School of Medicine, University of Pennsylvania, Philadelphia, PA, \\ USA
}

\begin{abstract}
Adolescence is a time period in development when the brain undergoes substantial remodeling in response to the environment. To determine whether a stressful experience during adolescence affects adult behavior, we exposed adolescent male and female C57BL/6) mice to chronic unpredictable stress (CUS) for 12 days starting at postnatal day 28 (PND28). We also exposed adult male and female mice to CUS for 12 days beginning at PND70 to determine whether adolescence is a sensitive time period when stress can have longlasting effects on behavior. Regardless of when mice were exposed to stress, they were all tested exactly 30 days later in the marble burying task, elevated zero maze, acoustic startle response, and forced swim test. Adolescent stress exposure increased anxiety-like behaviors in adult male and female mice and decreased acoustic startle response in a sex-dependent manner. However, adult stress exposure did not change anxiety or response to an acoustic tone in adult male or female mice as compared with nonstressed animals. Of interest, increased depression-like behavior in the forced swim test was observed in all mice, regardless of when the stress occurred. Gene expression analysis showed significant upregulation of corticotropin releasing factor receptor 2 (CrfR2) in the amygdala of males subjected to CUS during adolescence, but not in males that experienced CUS during adulthood. In contrast, females, regardless of when they were exposed to CUS, were not affected. These data support clinical evidence suggesting that early-life stress may predispose individuals to increased anxiety and depression later in life.

Neuropsychopharmacology (2017) 42, 1670-1678; doi:10.1038/npp.2017.1 I; published online I February 2017
\end{abstract}

\section{INTRODUCTION}

Adolescence is a dynamic time period for growth and development. The developing nervous system in particular undergoes heightened remodeling in response to the environment (Spear, 2000). During this time, synapse overproduction and pruning (Teicher et al, 1995), as well as transient changes in neurotransmitter and receptor production, facilitate neuronal maturation (WhitakerAzmitia, 1991). However, extreme early-life adversity may interrupt some of these processes, predisposing an individual to neuropsychiatric disorders and maladaptative behavior later in life (Andersen and Teicher, 2004). The onset of anxiety, depression, and posttraumatic stress disorders have been correlated with exposure to stressors during adolescence (Pelcovitz et al, 1994; Turner and Lloyd, 2004). Specifically, exposure to uncontrollable adverse events throughout childhood is linked to future psychiatric

*Correspondence: Dr JA Blendy, Department of Systems Pharmacology and Translational Therapeutics, University of Pennsylvania, Translational Research Laboratory, 125 South 31st Street, Philadelphia, PA 19104, USA, Tel: 215898 0730, Fax: 215573 2236,

E-mail: blendy@mail.med.upenn.edu

Received II July 20 16; revised I4 December 20 16; accepted 2 January 2017; accepted article preview online 17 January 2017 disorders and adolescent stressors are additive in predicting development and persistence of psychiatric events later in life (Kessler et al, 1997). Thus, clinical data suggest that early-life stress that is chronic and uncontrollable constitutes a major risk factor for the development of mental disorders.

A key brain region that mediates central stress response, the amygdala, undergoes significant remodeling through synapse overproduction and pruning during adolescence and is particularly sensitive to early-life stress exposure (Andersen and Teicher, 2004). Maladaptation of stress signaling, specifically altered expression of corticotropin releasing factor ( $\mathrm{Crf}$ ) has been found in the amygdala of rodents exposed to chronic adolescent stress (Plotsky et al, 2005) and CRF signaling in the limbic system mediates control of anxiety (Wiersma et al, 1995), stress (Bale et al, 2002), and startle response (Dirks et al, 2002). In addition to the amygdala, the bed nucleus of the stria terminalis (BNST) mediates response to stressful events via a vast network of projections throughout the central nervous system. Importantly, the BNST has been implicated in sustained stress response and anxiety through CRF-mediated interactions with the amygdala (Davis et al, 2010) and independently mediates startle response (Walker and Davis, 1997).

Animal studies modeling the effects of adolescent stress on behavior later in life suggest that exposure to early-life 
adversity facilitates differential responses to stressful situations during adulthood, such as enhanced anxiety and stress sensitivity. Chronic exposure to physical, social, or a combination of physical and social stressors during juvenile and prepubescent phases of development results in altered cognition, anxiety, and depression in adulthood in mice (Schmidt et al, 2010; Weiss et al, 2011; Saavedra-Rodríguez and Feig, 2013) and rats (Tsoory et al, 2007; McCormick et al, 2008; Eiland et al, 2012). Although these studies provide evidence for the long-lasting impact of adolescent stress, few studies have conducted a direct comparison of the effects of stress on behavior and neurobiology when experienced by the adolescent $v s$ the adult.

We determined the long-term effects of chronic stress on anxiety, startle response, and depression-like behavior in animals using a chronic unpredictable stress (CUS) paradigm. This paradigm is composed of both physical and social stressors that last 12 days and produces anhedonia and depression phenotypes in mice (Schmidt and Duman, 2010). The use of CUS in rodents mirrors uncontrolled stress experienced by individuals (Hollis et al, 2013) and prevents habituation to stress exposure, thus avoiding attenuated responses (Girotti et al, 2006). Mice were exposed to the same CUS during different developmental time windows, adolescence before puberty (postnatal day (PND) 28-40) or adulthood (PND70-82), and tested during adulthood (30 days following the last day of the stressor). By isolating the time period of stress exposure, we identified adolescence as a sensitive window for CUS to have long-term effects on behavior and gene expression.

\section{MATERIALS AND METHODS}

\section{Animals}

Male and Female C57BL/6JTac mice (6-8 weeks of age, 20-30 g) were ordered from Taconic Farms (Hudson, NY), group housed, and maintained on a $12 \mathrm{~h}$ light/dark cycle with food and water ad libitum in accordance with the University of Pennsylvania Animal Care and Use Committee (Philadelphia, PA). Mice were bred for two generations to generate offspring used in the current study. Breeding within the facility decreased the impact of transportation stress on the mice and allowed us to isolate the effects of the exposure of CUS in adolescents and adults. All experimental testing sessions were conducted between $0800 \mathrm{~h}$ and $1700 \mathrm{~h}$, with animals randomly assigned to treatment conditions. Because litters were born at varying times throughout the course of these studies, three different cohorts of mice were used for adolescent CUS exposure, whereas one cohort of mice was used for adult exposure. Separate cohorts of mice were used to determine changes in gene expression following stress exposures.

\section{Chronic Unpredictable Stress}

Mice underwent CUS for 12 days during the light phase of the $24 \mathrm{~h}$ period starting at PND28 or PND70 (4 weeks or 10 weeks of age). The CUS paradigm was adapted from previous studies (Schmidt and Duman, 2010). The exact stressors can be found in Supplementary Table S1. Briefly, animals were exposed to three stressors each day, in the morning, afternoon, and overnight, for 12 consecutive days in dedicated procedure rooms. Mice were returned to the animal colony between stressors and after the final stressor. Three cohorts of adolescent and one cohort of adult mice underwent CUS, sucrose preference testing, and behavioral testing. To determine whether sex is a major contributor in the efficacy of the CUS to induce anhedonia, we exposed an additional cohort of mice to CUS and evaluated sucrose preference.

\section{Sucrose Preference Test (SPT)}

Sucrose consumption was evaluated on the last 2 days of the CUS exposure to determine the consequence of these stressors on sucrose preference in the animals. Mice were habituated to a $1 \%$ sucrose solution for $48 \mathrm{~h}$ starting on day 6 of CUS exposure to prevent neophobia during testing. Following sucrose exposure, increasing water restriction was used to habituate animals to water restriction: water was restricted for $4 \mathrm{~h}$ on day $8,14 \mathrm{~h}$ on day 9 , and $19 \mathrm{~h}$ on day 10 . On day 11, mice were allowed access to sucrose solution in a cage filled with home cage bedding for $1 \mathrm{~h}$ without the presence of cage mates. Testing was repeated on day 12 of CUS exposure except that mice were given access to water instead of the sucrose solution. Sucrose preference was reported as the difference between total sucrose consumption divided by total liquid consumption (ml) on both test days.

\section{Behavioral Tests}

Behavioral testing was conducted 30 days following the last day of CUS exposure during the light phase of the $24 \mathrm{~h}$ period: PND70-82 (10-12 weeks of age) for the adolescent stress exposure group or PND112-126 (16-18 weeks of age) for the adult stress exposure group (Figure 1a). Behavior was assayed in the following sequential order in every animal: (1) marble burying (MB) task, (2) elevated zero maze (EZM), (3) acoustic startle response (ASR), and (4) forced swim test (FST), with a period of rest of at least 2 days between each test. Previous studies demonstrate that order of testing affects behavioral measurements. Therefore, all animals were tested in the same order, and FST was the final behavioral assay to be administered (Wahlsten, 2010). Immediately following the FST, animals were killed and whole brains were rapidly removed and flash frozen in isopentane $\left(-80^{\circ} \mathrm{C}\right)$.

\section{Marble Burying}

Differences in anxiety between treatment groups were evaluated using the MB test (Jimenez-Gomez et al, 2011). After a $1 \mathrm{~h}$ period of acclimation, mice were placed individually in a test cage that resembled their home cage $(26 \times 20 \times 14 \mathrm{~cm})$. Twenty marbles were distributed evenly in the cages in 5 rows of 4 on top of mouse bedding $(5 \mathrm{~cm}$ in depth), and a clear lid was placed on top of the cage. Animals were left undisturbed for $15 \mathrm{~min}$, after which time the number of marbles buried, distinguished by being threefourths or more submerged under bedding, was quantified by a trained observer blind to experimental groups. 

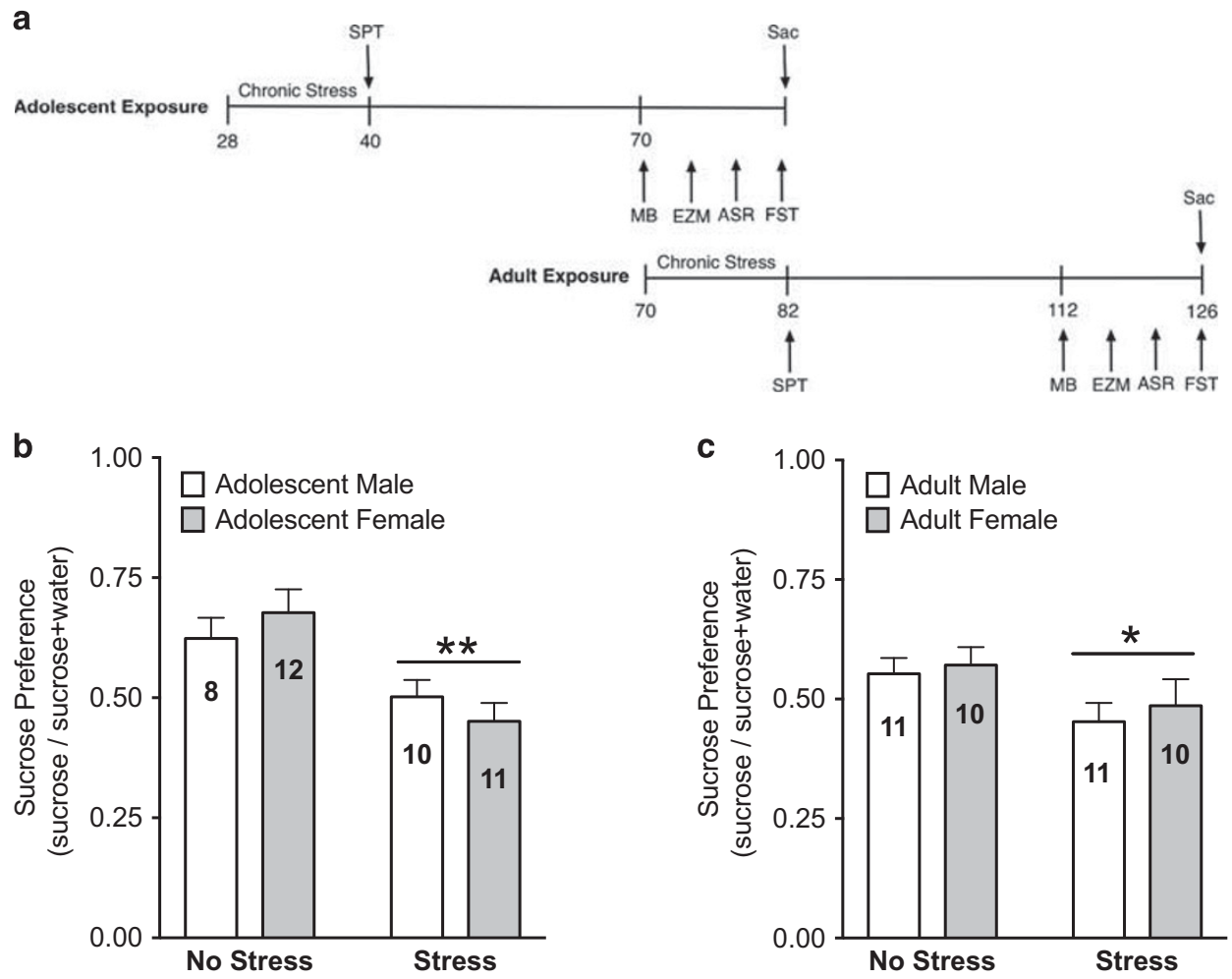

Figure I Experimental timeline and sucrose preference test immediately following stress exposure. (a) Experimental schematic for chronic stress exposure during adolescence and adulthood and behavioral testing. Male and female mice underwent 12 days of CUS during adolescence, PND28-40, or adulthood, PND70-82. A sucrose preference test (SPT) was conducted on the final two days of CUS exposure in both groups. At 4 weeks following the final day of CUS exposure, beginning on PND70 or PNDI 12 respectively, animals were tested in the marble burying (MB) task, elevated zero maze (EZM), acoustic startle response (ASR), and forced swim test (FST) with at least 2 days of rest between behavioral testing. Animals were killed (SAC) and whole brains were removed and frozen immediately following FST. (b) Adolescent exposure to stress (Stress) decreases preference for sucrose over water in male and female mice compared with controls (No Stress) $(* * P<0.00 \mathrm{I})$. Bars represent volume of sucrose consumed divided by total volume of water and sucrose consumed \pm SEM. Sample sizes of each condition are reported in the figure. (c) Adult CUS exposure decreases preference for sucrose over water in mice $(* P<0.05)$.

\section{Elevated Zero Maze}

The EZM was used as a second test of anxiety. Following a $1 \mathrm{~h}$ period of acclimation to the testing room, mice were placed in the maze consisting of two open arms and two closed arms elevated 24 inches off the ground and left undisturbed for $5 \mathrm{~min}$. Mice were video recorded for the duration of testing. Time spent in the open arms of the maze was measured by a trained observer blind to experimental groups.

\section{Acoustic Startle Response}

The reflexive response to an unexpected tone was assessed using the ASR (Davis, 1980). After a $1 \mathrm{~h}$ period of acclimation to the testing room, animals were placed in acoustic startle chambers (SR-Labs, San Diego, CA) for behavioral testing. The chamber consisted of a light- and sound-attenuating outer plastic box and an inner nonrestrictive plastic cylinder chamber affixed to a stage platform. Broadband acoustic startle tones were emitted from a highfrequency speaker mounted above the mouse chamber. Startle reflexes were measured by a piezo electronics monitor mounted under the stage platform. Each testing session lasted $30 \mathrm{~min}$. Animals were habituated to the inside of the startle chamber with a $67 \mathrm{~dB}$ sound pressure level (SPL) background white noise for $5 \mathrm{~min}$. After the habituation period, animals were presented with 10 rounds of 5 pseudorandom startle tones (50 total trials) differing in $\mathrm{dB}$ SPL $(75,80,85,90,95,100105,100,115$, and $120 \mathrm{~dB}$ SPL). Pseudo random interstimulus intervals (ISIs) were generated by the Startle Response software (SR-Labs). ISIs consisted of $26,28,30,32$, and $34 \mathrm{~s}$. Immediately after each startle tone presentation, the startle amplitude was measured as the average voltage emitted by the piezo electric pickup each millisecond for the $100 \mathrm{~ms}$ response window.

\section{Forced Swim Test}

Immobility differences between treatment groups were evaluated using the FST. Mice were placed into plexiglas cylinders filled with water $\left(25^{\circ} \mathrm{C} ; 30-38 \mathrm{~cm}\right.$ high $)$ for $6 \mathrm{~min}$, and behavior was video recorded. The time spent immobile during the swim session was recorded by an observer blinded to treatment groups. A mouse was considered immobile when making only those movements necessary to keep its head above water.

\section{RNA Extraction, cDNA Synthesis, and Quantitative Real-Time PCR}

Coronal brain sections $(300 \mu \mathrm{m})$ between Bregma -0.58 and -1.70 for the amygdala, 0.26 and -0.34 for the BNST, 
and 2.96 and 1.94 for the prefrontal cortex (PFC) (Franklin and Paxinos, 1997) were used to collect $1.2 \mathrm{~mm}$ punches bilaterally of each brain region. RNA was extracted by homogenizing in $800 \mu \mathrm{l}$ of TRIzol and $160 \mu \mathrm{l}$ of chloroform. RNA was purified using an RNeasy Mini Kit (Qiagen, Cat. No. 74104). RNA concentration and integrity were determined with a Nanodrop spectrophotometer (Nanodrop Technologies, Wilmington, DE). cDNA was synthesized from RNA (100 ng) using the High-Capacity cDNA Reverse Transcription Kit (Applied Biosystems). Quantitative realtime PCR (qRT-PCR) was carried out using the SYBR-green master mix (Applied Biosystems) and $10 \mu \mathrm{M}$ primers (final concentration) for corticotropin relating factor (Crf) and corticotropin releasing factor receptors 1 and 2 (CrfR1, CrfR2) on the Stratagen MX3000 using MXPro QPCR software. Primer sequences for Crf, CrfR1, CrfR2, and the housekeepers Tbp and Hprt can be found in Supplementary Table S2. Cycling parameters were $95^{\circ} \mathrm{C}$ for $10 \mathrm{~min}$ and then 40
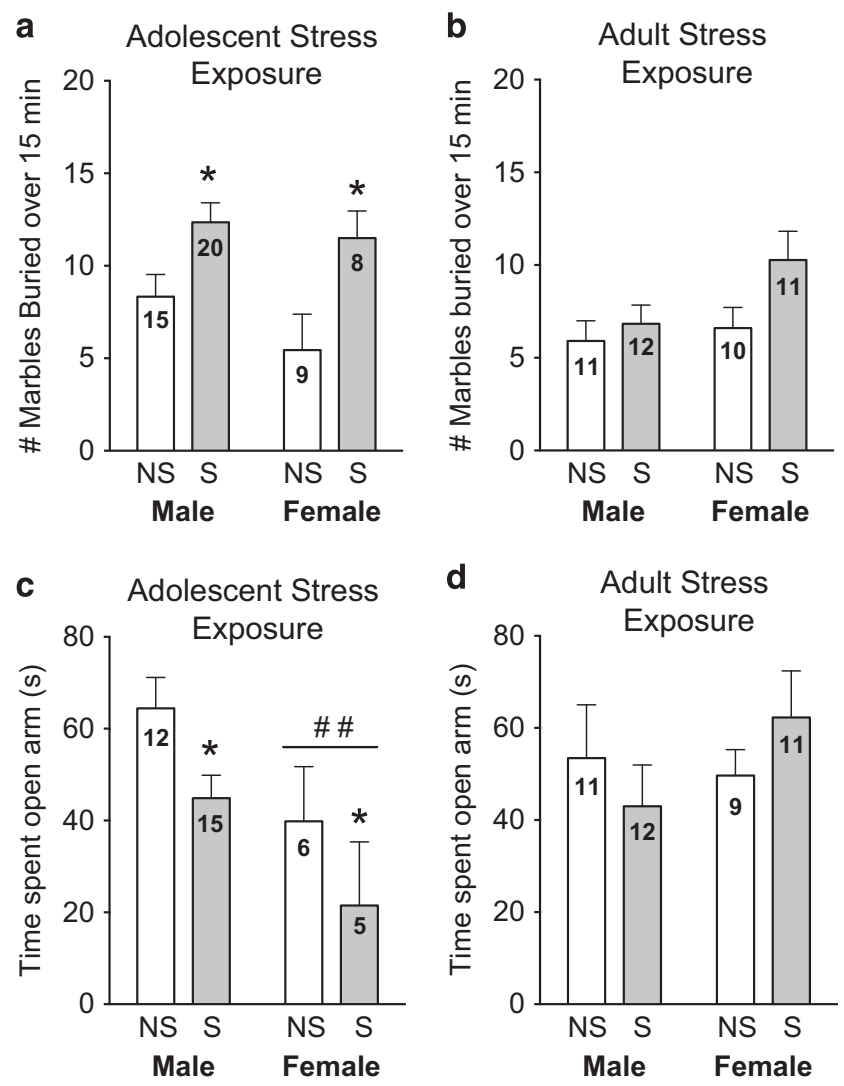

Figure 2 Adolescent CUS increases anxiety-like behaviors in adulthood. (a) Male and female mice exposed to adolescent CUS (S) buried more marbles in the MB task in adulthood than nonstressed (NS) control animals $(* P<0.05)$. Bars represent number of marbles buried \pm SEM. Sample sizes are reported in the figure. (b) There is no difference in number of marbles buried between animals exposed to adult CUS and nonstressed control animals later in adulthood. (c) Male and female mice exposed to adolescent CUS spend less time in the open arm of the EZM as compared with NS controls ( $P<0.05)$. Female mice spent less time in the open arm of the EZM regardless of adolescent stress exposure compared with male mice $\left({ }^{\# \#} P<0.0 I\right)$. Bars represent time spent in the open arm of the EZM in seconds \pm SEM. (d) There is no difference in the amount of time spent in the open of the EZM in male and female mice exposed to adult CUS compared with nonstressed controls. cycles of $95^{\circ} \mathrm{C}(30 \mathrm{~s})$ and $60^{\circ} \mathrm{C}(1 \mathrm{~min})$, followed by a melting curve analysis. All reactions were run in triplicate, and median cycles to threshold (Ct) values were used for analysis. Housekeeping genes were used to normalize against experimental genes, and relative gene expression was determined using the $2^{-\Delta \Delta C T}$ method (Livak and Schmittgen, 2001).

\section{Statistical Analysis}

All data are presented as mean \pm SEM. For comparisons of stress effects among both sexes in each behavior (eg, SPT, $\mathrm{MB}, \mathrm{EZM}, \mathrm{FST}$ ), a two-way analysis of variance (ANOVA) was used. For comparisons between multiple groups at multiple measurement points (eg, ASR), a repeated measures two-way ANOVA (RM-ANOVA) was used to determine significant differences with tone representing the within, repeated-measures independent factor and stress exposure the dependent variable with sexes analyzed independently. Finally, for comparisons between experimental groups with age and stress as independent variables (eg, mRNA fold change), a two-way ANOVA was used. Statistical analyses were performed using Graphpad Prism 7 (Graphpad Software, La Jolla, CA), with the threshold for statistical significance set as $P<0.05$, and Bonferroni multiple comparison test used for all post hoc analysis.

\section{RESULTS}

\section{Loss of Sucrose Preference Immediately Following CUS Exposure in Adolescents and Adults}

Mice exposed to CUS had a significantly lower preference score in the sucrose preference test when tested on the last day of stress exposure as compared with within-group control animals not exposed to adolescent or adult stress (Figure 1b; $\mathrm{F}_{1,37}=16.35, P<0.001$, Figure 1c; $\mathrm{F}_{1,38}=0.3801$, $P<0.05)$. In addition, a two-way ANOVA revealed a significant effect of age of testing. Animals exposed to CUS in adulthood (PND82) had a significantly lower preference score compared with animals exposed to CUS during adolescence $(\mathrm{PND} 40)\left(\mathrm{F}_{1,54}=8.788, P<0.01\right)$.

\section{Adolescent CUS Increases Anxiety-Like Behaviors in Adulthood}

Exposure to CUS during adolescence increased the number of marbles buried in the MB task by adult mice compared with nonstressed controls (Figure 2a; $\mathrm{F}_{1,48}=12.41$, $P=0.0009)$. However, mice exposed to CUS during adulthood and tested 30 days later did not bury more marbles than nonstressed controls (Figure 2b; $\mathrm{F}_{1,40}=3.622$, $P=0.0642$ ). In a second test of anxiety, the EZM, adult mice exposed to adolescent CUS spent less time in the open arm of the maze compared with nonstress controls tested at the same time and age (Figure $2 \mathrm{c} ; \mathrm{F}_{1,33}=4.707, P<0.05$ ). A twoway ANOVA also revealed that females, regardless of stress exposure, spent less time in the open arms of the elevated zero maze when tested in adulthood (10 weeks) compared with males (Figure $2 \mathrm{c} ; \mathrm{F}_{1,33}=7.541, P<0.01$ ). In contrast, CUS exposure during adulthood had no effect on anxiety behavior in the EZM in male or female mice (Figure 2d; 

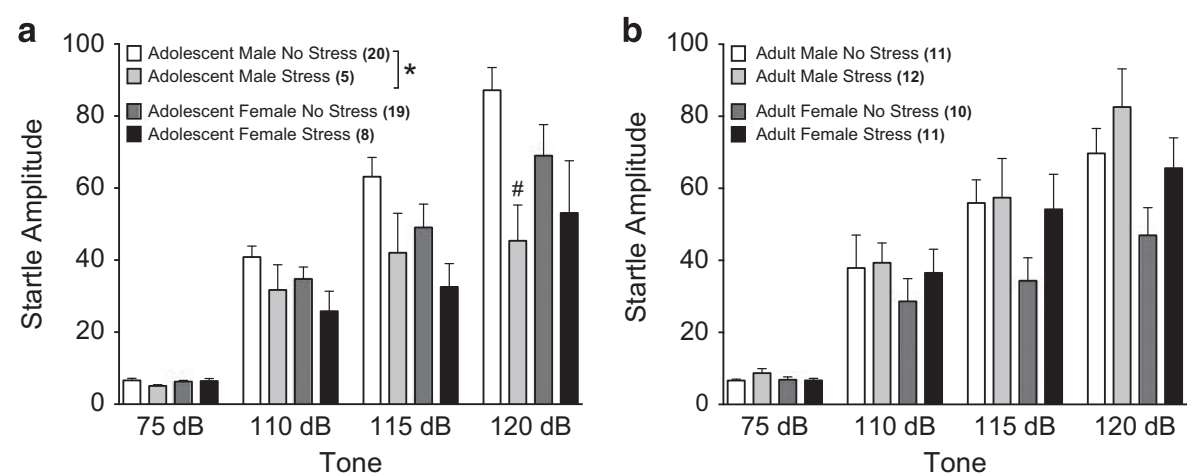

Figure 3 Adolescent CUS decreases acoustic startle response in male mice in adulthood. (a) Exposure to adolescent CUS decreases startle amplitude at high $\mathrm{dB}$ tones in male adult mice compared with nonstressed controls $(* P<0.05)$. Values are plotted as startle amplitude \pm SEM. Sample sizes of each condition are reported in the figure. The lowest $\mathrm{dB}$ SPL and three highest db SPL tones are shown. Multiple comparisons revealed a significant difference between adolescent male no stress and adolescent male stress at I $20 \mathrm{~dB}$ SPL $\left({ }^{\#} P<0.00 \mathrm{I}\right)$. Female mice exposed to adolescent CUS showed no difference in startle response compared with nonstressed controls. (b) Male and female mice exposed to adult CUS showed no difference in startle response compared with nonstressed controls. Additional statistical analysis showed no significant differences between sexes at any dB examined.

\section{Adolescent Stress Exposure}
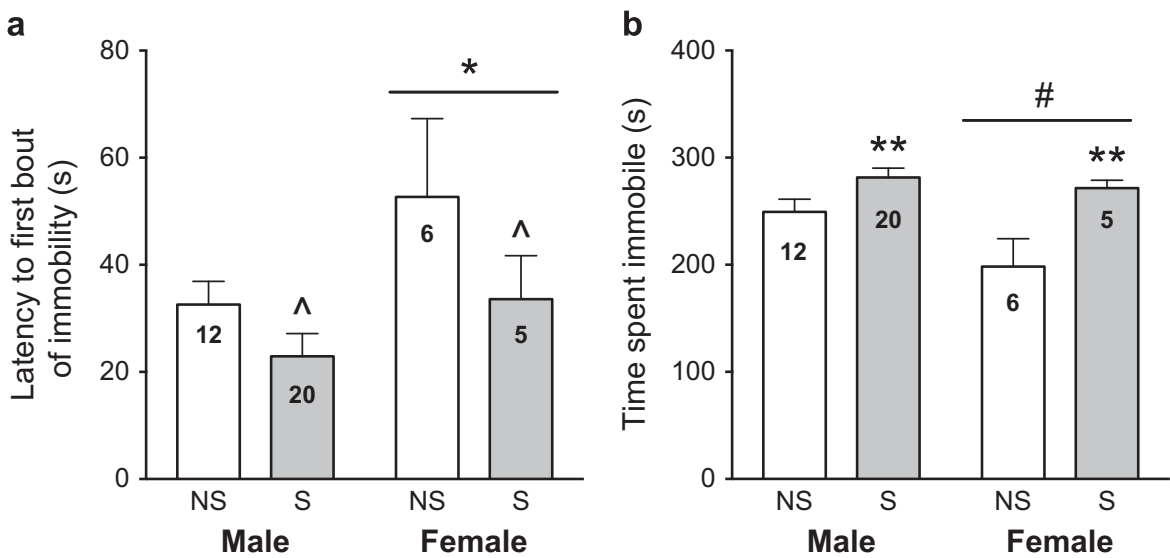

Adult Stress Exposure
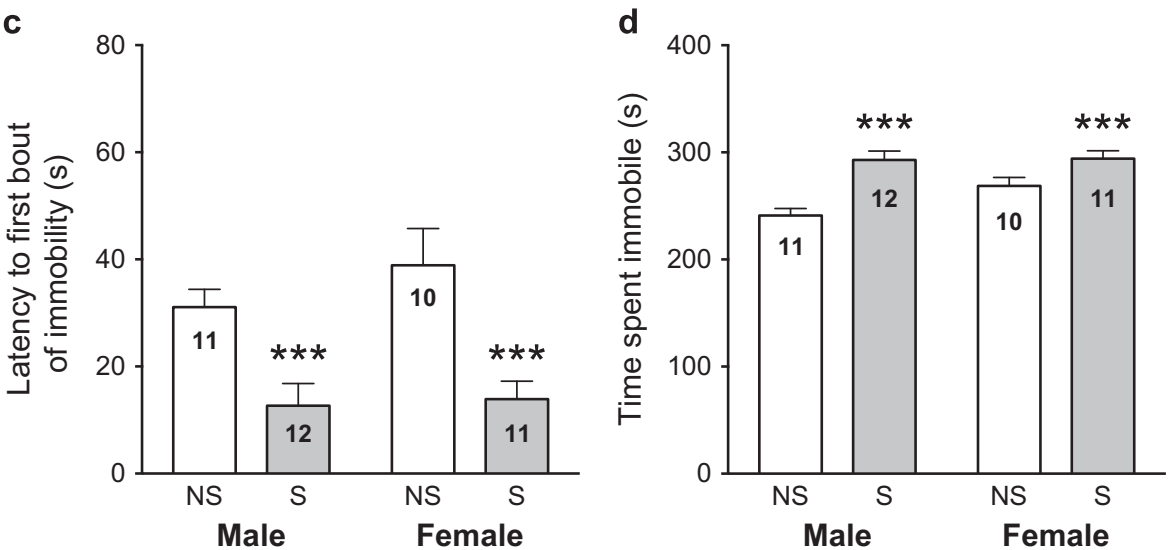

Figure 4 Adolescent and adult CUS induces depression-like phenotype in mice in adulthood. (a) A main effect of sex $(* P<0.05)$ and a trend toward a decrease in latency to first bout of immobility due to a main effect of stress ( $P P=0.0596)$ was found in male and female mice exposed to adolescent stress $(S)$ and tested in the FST in adulthood compared with no stress controls (NS). Bars represent latency in seconds to first bout of immobility \pm SEM. Sample sizes are indicated on the figure. (b) Male and female adult mice exposed to adolescent CUS spend more time immobile in the FST compared with nonstressed control male and female mice $(* * P<0.001)$. A main effect of sex influenced time spent immobile in the FST in male and female mice $\left({ }^{\#} P<0.05\right)$. Bars represent time spent immobile in seconds over 6 consecutive min \pm SEM. (c) Exposure to adult CUS decreases latency to first bout of immobility in male and female mice compared with nonstress controls $(* * * *<0.000 \mathrm{I})$. (d) Male and female adult mice exposed to adult CUS spend more time immobile over the 6 min FST compared with nonstressed controls (****P $<0.000$ I). 
$\left.\mathrm{F}_{1,39}=0.01236, P=0.9120\right)$ and no differences were found between sexes.

\section{Adolescent CUS Decreases Acoustic Startle Response at High Decibel Tones in a Sex-Dependent Manner}

Exposure to adolescent CUS decreased average startle response at high $\mathrm{dB}$ tones in male mice. A two-way RM-ANOVA revealed a main effect of tone $\left(F_{3,69}=46.72\right.$, $P<0.0001)$ and a main effect of adolescent stress exposure $\left(\mathrm{F}_{1,23}=6.573, P=0.0174\right)$ along with a tone by stress exposure interaction $\left(\mathrm{F}_{3,69}=5.332, P=0.0023\right)$ in male mice
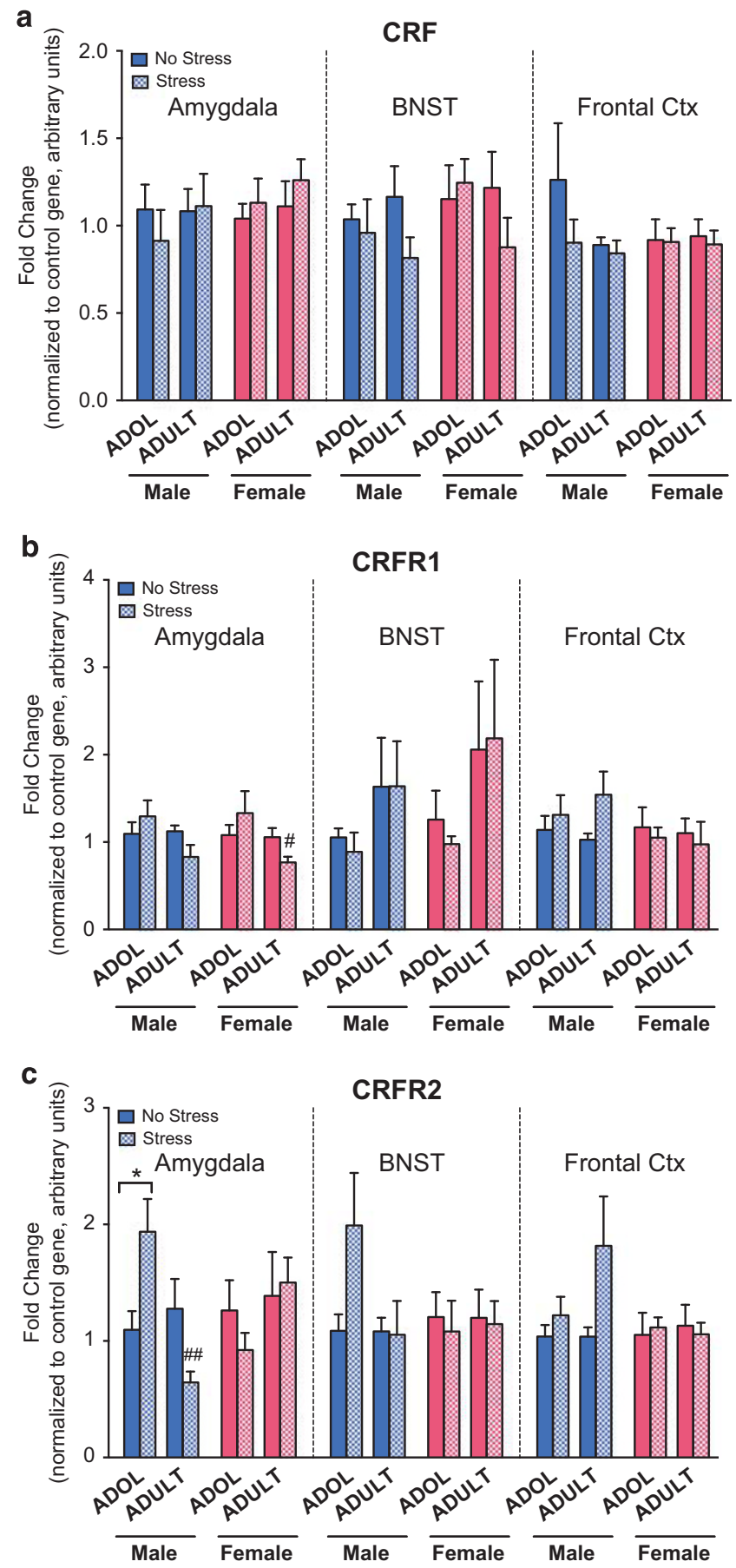

on the ASR task (Figure 3a). Multiple comparisons further revealed a significant effect of adolescent stress on startle response at the highest tone administered, $120 \mathrm{~dB}$ SPL $(P<0.0001)$. In contrast, CUS exposure during adulthood showed no significant change in startle response at high $\mathrm{dB}$ tones when compared with nonstressed controls in males (Figure 3a; $F_{1,21}=0.3038, P=0.5873$ ). However, as expected, there was a main effect of startle tone on startle response amplitude $\left(\mathrm{F}_{3,63}=55.59, P<0.0001\right)$.

There was no change in startle response because of adolescent CUS exposure in female mice tested for startle response in adulthood $\left(\mathrm{F}_{1,25}=3.424, P=0.0761\right)$, but, as expected, there was a main effect of tone across both treatment groups (Figure $3 \mathrm{~b} ; \mathrm{F}_{3,75}=31.17, P<0.0001$ ). Furthermore, female mice exposed to adult CUS showed no significant changes in startle responses compared with nonstressed controls (Figure $3 \mathrm{~b} ; \mathrm{F}_{1,19}=2.491, P=0.1310$ ), but did show an expected effect of tone on startle amplitude $\left(\mathrm{F}_{3,57}=37.31, P<0.0001\right)$.

\section{Adolescent and Adult CUS Increases Time Spent Immobile in the FST}

Exposure to CUS increased total time spent immobile in the FST and decreased latency to the first bout of immobility in both male and female mice, regardless of when stress was presented. A two-way ANOVA revealed that male and female mice exposed to adolescent CUS and tested 30 days later have a trend toward a shorter latency to the first bout of immobility (Figure 4a; $\mathrm{F}_{1,39}=3.765 ; P=0.0596$ ). In addition, there is significant increase in latency to the first bout of immobility in female mice regardless of adolescent stress exposure compared with male mice tested at the same age (12 weeks; $\mathrm{F}_{1,39}=4.317, P<0.05$ ). In addition, both male and female mice exposed to adolescent CUS spent significantly more time immobile compared with nonstressed controls (Figure $4 \mathrm{~b} ; \mathrm{F}_{1,39}=4.257, P<0.05$ ) with an additional main effect of sex driven by less time spent immobile by control females compared with males $\left(\mathrm{F}_{1,39}=12.7\right.$, $P<0.01)$. Male and female mice exposed to CUS during adulthood and tested later in adulthood (18 weeks) showed a decreased latency to the first bout of immobility (Figure 4c;

Figure 5 Quantitative real-time PCR analysis of $\mathrm{Cr}$, $\mathrm{Cr} R \mathrm{RI}$, and $\mathrm{Cr} R \mathrm{R} 2$ expression in the amygdala, bed nucleus of the stria terminalis (BNST), and prefrontal cortex of stressed mice shows increased CrfR2 in male mice exposed to adolescent CUS. (a) Male and female mice exposed to adolescent or adult stress (Stress) have no fold change in Crf mRNA expression in the amygdala, BNST, or prefrontal cortex compared with controls (No Stress). Bars represent fold change measured by qRT-PCR and normalized to Tbp for Crf or HPRT for CrfRI and CrfR2 \pm SEM; $n=9-13$. (b) Stress had no effect on CrfRI expression in the amygdala, BNST, or frontal cortex of male mice. Adult female mice exposed to adult CUS showed a significant decrease in $\mathrm{Cr} R \mathrm{R} /$ expression in the amygdala compared with females exposed to adolescent CUS $\left({ }^{\#} P<0.05\right)$, whereas no significant differences were found in other brain regions. (c) Males exposed to adolescent CUS showed a significant increase in CrfR2 expression in the amygdala compared with nonstressed controls $(* P<0.05)$ and males exposed to adult CUS had a significant decrease in CrfR2 expression compared with males exposed to adolescent CUS ( $\left.{ }^{\#} P<0.001\right)$. No significant changes were found in other brain regions in CrfR2 expression in male mice or in female mice. 
$\left.\mathrm{F}_{1,40}=23.04, P<0.0001\right)$ and spent more time immobile (Figure 4d; $F_{1,40}=25.7, P<0.0001$ ) compared with controls, but there was no effect of sex. It should be noted that there was a significant effect of age in the no stress group on time spent immobile; female controls tested in the forced swim at 18 weeks spent more time immobile compared with females tested at 12 weeks of age $\left(t_{14}=3.179, P<0.01\right)$.

\section{Adolescent CUS Increases CrfR2 in Adult Male Mice}

To evaluate molecular changes associated with anxiety and startle reaction, we examined gene expression in the amygdala, BNST, and prefrontal cortex of the adult mice. Crf expression was unaltered in the amygdala, BNST, or prefrontal cortex of male or female animals exposed to either adolescent or adult CUS (Figure 5a). In addition, CrfR1 mRNA levels were not altered by stress in male or female mice in any brain region (Figure 5b). However, it should be noted that there was a main effect of age on the expression of CrfR1 in adult female mice in the amygdala $\left(F_{1,45}=4.203\right.$, $P<0.05)$. Of interest, adolescent CUS exposure but not adult exposure increased expression of CrfR2 mRNA in the amygdala of adult males (main effect of age: $\mathrm{F}_{1,44}=7.048$, $P<0.05$ and interaction: $\mathrm{F}_{1,44}=12.37, P<0.001$; Figure $5 \mathrm{c}$ ), whereas the same was not found in other brain regions examined or in female mice.

\section{DISCUSSION}

Early-life stress may predispose individuals to neuropsychiatric disorders later in life. Childhood trauma is associated with the emergence of stress-related pathologies in adulthood that include depression, anxiety, and posttraumatic stress disorder (Moffitt et al, 2007). In addition, quality of life and coping psychopathology is heavily influenced by experiences during adolescence (Neigh et al, 2009).

We investigated whether or not adolescence represents a window of development that is sensitive to stress exposure. Previous work has identified effects of adolescent stress exposure on adult behavior in mice (Saavedra-Rodríguez and Feig, 2013; Weiss et al, 2011). However, previous studies did not directly compare the effects of the same type of stressors, in both duration and intensity, during adolescence and adulthood. Furthermore, we tested for changes in behavior exactly 30 days following the final day of stress exposure, Thus, in this experimental paradigm we controlled for the type of stress experienced as well as the incubation period between stress and behavior testing. In addition, we used a stress paradigm composed of physical and emotional stressors and one that is both chronic and unpredictable in nature to better model the human experience of stress (Hill et al, 2012).

Approximately 30 days following the end of CUS exposure, stress-exposed male and female mice spent more time immobile in the FST. Conflicting studies report increases and decreases in immobility (depression and antidepressantlike behaviors, respectively) when animals experience stress in adolescence or adulthood (for review see Hollis et al, 2013). Our data demonstrate that with CUS a consistent depression-like phenotype persists in male and female adults regardless of when the stress was administered, in adolescence or adulthood. Although age did not play a role in performance on this task for males, we did find an age difference (12 vs 18 weeks) in time spent immobile between controls in female mice. Thus, the difference between the stress and no stress group may not reflect an increase in mobility in the stressed females but instead a decrease in mobility due to age. As stress exposure still increased immobility in female mice compared with their age-matched controls at 18 weeks, it is likely this is a real effect of prior stress exposure. Although the depression phenotype was affected in all animals regardless of time of stress exposure, we note that this is in contrast to CUS affects on anxiety behaviors that are affected only if stress occurs during adolescence. It is unclear as to why CUS affected depressionlike behavior in both ages, but affected more anxiety-related measures when occurring in adolescents. This difference may be because of sensitivity to stress in developing brain areas associated with these distinct behavioral domains (Eiland et al, 2012).

Measurement of anxiety-like behavior in rodents comes with peculiarities and limitations, and no one test provides the ideal model of anxiety (Steimer, 2011). We chose two paradigms that measure different aspects of anxiety. The MB test measures active and compulsive anxiety behavior (Jimenez-Gomez et al, 2011), whereas the EZM measures exploratory behavior in a circular maze (Shepherd et al, 1994). Adolescent CUS exposure increased anxiety-like behavior in both of these tests. Of importance for clinical translation, a predisposition toward an anxious temperament promotes depression and anxiety diagnosis in patients (Nyman et al, 2011). In contrast, adult CUS-exposed mice showed no change in anxiety behavior. Of note, control and CUS-exposed female mice showed greater anxiety behavior in the EZM compared with males. This sex-specific effect has been previously characterized (Dalla et al, 2010) and appears to be a product of age (Walf et al, 2009) and estrous cycle (Gouveia et al, 2004).

To characterize additional stress-relevant behaviors following CUS, we examined startle response to an acoustic tone. We found adolescent CUS decreased startle amplitudes in males, but not females. Because of hormonal differences, males and females are predisposed to experience the effects of stress differently. In fact, programming of limbic stress circuitry and sex-specific responses to stress later in life occurs before puberty and is dependent on intricate sexspecific hormone signaling (Bale et al, 2010). Some studies found female rodents to be resilient to the effects of stress, unless ovariectomized (Galea et al, 1997), whereas others characterized females to be more vulnerable to the effects of stress (Mueller and Bale, 2008). Further research into sexdependent effects of chronic stress will continue to promote a better understanding of stress-dependent changes in behavior.

Increased anxiety- and sex-specific effects in startle response following adolescent but not adult stress may be related to the vulnerability of the developing extrahypothalamic stress pathway in the amygdala and extended amygdala (BNST) during adolescence (Lupien et al, 2009). Indeed, anxiety and startle response behaviors, as opposed to depression, were particularly sensitive to adolescent stress, possibly because of ongoing amygdalar development in adolescence (Koss et al, 2014). In the amygdala, CRF 
mediates extrahypothalamic stress response and is implicated in both anxiety and depression disorders. Increased CRF activity in the amygdala is found immediately following chronic stress (Menzaghi et al, 1993), and increases in CRF signaling in the amygdala increases anxiety in rats (Rainnie et al, 2004).

Crf expression and signaling has also been implicated in ASR behavior in rodents. CRF projections from the amygdala to the pontine reticular nucleus mediate startle response in rodents (Davis, 1980), and stimulation of the amygdala (Koch and Ebert, 1993) or pharmacologic increase of CRF signaling enhances startle amplitudes Thus, our findings that $C r f R 2$ was increased in the amygdala suggests that stress exposure may mediate prolonged changes in CRF signaling in the central nervous system. These changes in gene expression occur only when stress is presented during adolescence as no comparable differences were observed in mice that were exposed to CUS during adulthood.

CRFR2 is found predominantly in the medial and posterior nuclei (Chalmers et al, 1995) and is distributed both preand postsynaptically in the amygdala (Treweek et al, 2009). Activation of CRFR2, by either CRF or urocortin, mediates stress coping through dampening stress sensitivity (Bale et al, 2002). Therefore, increased CrfR 2 expression in the amygdala may contribute to blunted startle response as well as heightened anxiety found in male mice exposed to adolescent stress, the latter supported by evidence that CRFR2 activation is anxiogenic (Reul, 2002). However, future studies need to correlate expression levels with receptor activation. It should be noted that these molecular changes were only found in males exposed to adolescent stress, and increased anxiety in females may be mediated through a different mechanism not explored in these studies.

In summary, evidence from these studies suggest that adolescence is a sensitive window for stress exposure, producing a disposition toward increased anxiety, depression, and altered reactivity to stress in the future. In addition, this characterization extends to both sexes and identified sexspecific differences in response to stress. Together, these data provide evidence that sex and timing of stress exposure are important variables that affect future adult behaviors. Although we show changes in gene expression for members of the CRF signaling pathway, future studies aimed at investigating causal relationships between these proteins and behavior affected by early stress exposure are warranted.

\section{FUNDING AND DISCLOSURE}

The authors declare no conflict of interest.

\section{ACKNOWLEDGMENTS}

This work was supported by grants from the National Institute on Drug Abuse (R01DA033646 (to JAB) and T32DA28874 (to NLY)).

\section{REFERENCES}

Andersen SL, Teicher MH (2004). Delayed effects of early stress on hippocampal development. Neuropsychopharmacology 29: 1988-1993.
Bale TL, Baram TZ, Brown AS, Goldstein JM, Insel TR, McCarthy MM et al (2010). Early life programming and neurodevelopmental disorders. Biol Psychiatry 68: 314-319.

Bale TL, Lee K-F, Vale WW (2002). The role of corticotropinreleasing factor receptors in stress and anxiety. Integr Comp Biol 42: 552-555.

Chalmers DT, Lovenberg TW, De Souza EB (1995). Localization of novel corticotropin-releasing factor receptor (CRF2) mRNA expression to specific subcortical nuclei in rat brain: comparison with CRF1 receptor mRNA expression. J Neurosci 15: 6340-6350.

Dalla C, Pitychoutis PM, Kokras N, Papadopoulou-Daifoti Z (2010). Sex differences in animal models of depression and antidepressant response. Basic Clin Pharmacol Toxicol 106: 226-233.

Davis M (1980). Neurochemical modulation of sensory-motor reactivity: acoustic and tactile startle reflexes. Neurosci Biobehav Rev 4: 241-263.

Davis M, Walker DL, Miles L, Grillon C (2010). Phasic vs sustained fear in rats and humans: role of the extended amygdala in fear vs anxiety. Neuropsychopharmacology 35: 105-135.

Dirks A, Groenink L, Schipholt MI, van der Gugten J, Hijzen TH, Geyer MA et al (2002). Reduced startle reactivity and plasticity in transgenic mice overexpressing corticotropin-releasing hormone. Biol Psychiatry 51: 583-590.

Eiland L, Ramroop J, Hill MN, Manley J, McEwen BS (2012). Chronic juvenile stress produces corticolimbic dendritic architectural remodeling and modulates emotional behavior in male and female rats. Psychoneuroendocrinology 37: 39-47.

Franklin KBJ, Paxinos G. The Mouse Brain in Stereotaxic Coordinates. Academic Press Inc: San Diego, CA, 1997.

Galea LAM, McEwen BS, Tanapat P, Deak T, Spencer RL, Dhabhar FS (1997). Sex differences in dendritic atrophy of CA3 pyramidal neurons in response to chronic restraint stress. Neuroscience 81: 689-697.

Girotti M, Pace TWW, Gaylord RI, Rubin BA, Herman JP, Spencer RL (2006). Habituation to repeated restraint stress is associated with lack of stress-induced c-fos expression in primary sensory processing areas of the rat brain. Neuroscience 138: 1067-1081.

Gouveia A, Santos dos UD, Felisbino FE, de Afonseca TL, Antunes G, Morato S (2004). Influence of the estrous cycle on the behavior of rats in the elevated T-maze. Behav Processes 67: 167-171.

Hill MN, Hellemans KGC, Verma P, Gorzalka BB, Weinberg J (2012). Neurobiology of chronic mild stress: parallels to major depression. Neurosci Biobehav Rev 36: 2085-2117.

Hollis F, Isgor C, Kabbaj M (2013). The consequences of adolescent chronic unpredictable stress exposure on brain and behavior. Neuroscience 249: 232-241.

Jimenez-Gomez C, Osentoski A, Woods JH (2011). Pharmacological evaluation of the adequacy of marble burying as an animal model of compulsion and/or anxiety. Behav Pharmacol 22: 711-713.

Kessler RC, Davis CG, Kendler KS (1997). Childhood adversity and adult psychiatric disorder in the US National Comorbidity Survey. Psychol Med 27: 1101-1119.

Koch M, Ebert U (1993). Enhancement of the acoustic startle response by stimulation of an excitatory pathway from the central amygdala/basal nucleus of Meynert to the pontine reticular formation. Exp Brain Res 93: 231-241.

Koss KJ, Hostinar CE, Donzella B, Gunnar MR (2014). Social deprivation and the HPA axis in early development. Psychoneuroendocrinology 50: 1-13.

Livak KJ, Schmittgen TD (2001). Analysis of relative gene expression data using real-time quantitative PCR and the 2 (-Delta Delta C(T)) Method. Methods 25: 402-408.

Lupien SJ, McEwen BS, Gunnar MR, Heim C (2009). Effects of stress throughout the lifespan on the brain, behaviour and cognition. Nat Rev Neurosci 10: 434-445.

McCormick CM, Smith C, Mathews IZ (2008). Effects of chronic social stress in adolescence on anxiety and neuroendocrine 
response to mild stress in male and female rats. Behav Brain Res 187: 228-238.

Menzaghi F, Heinrichs SC, Pich EM (1993). The role of limbic and hypothalamic corticotropin-releasing factor in behavioral responses to stress. Ann NY Acad Sci 697: 142-154.

Moffitt TE, Caspi A, Harrington H, Milne BJ, Melchior M, Goldberg D et al (2007). Generalized anxiety disorder and depression: childhood risk factors in a birth cohort followed to age 32. Psychol Med 37: 441-452.

Mueller BR, Bale TL (2008). Sex-specific programming of offspring emotionality after stress early in pregnancy. J Neurosci $\mathbf{2 8}$ : 9055-9065.

Neigh GN, Gillespie CF, Nemeroff CB (2009). The neurobiological toll of child abuse and neglect. Trauma Violence Abuse 10: 389-410.

Nyman E, Miettunen J, Freimer N, Joukamaa M, Mäki P, Ekelund J et al (2011). Impact of temperament on depression and anxiety symptoms and depressive disorder in a population-based birth cohort. J Affect Disord 131: 393-397.

Pelcovitz D, Kaplan S, Goldenberg B, Mandel F, Lehane J, Guarrera J (1994). Post-traumatic stress disorder in physically abused adolescents. J Am Acad Child Adolesc Psychiatry 33: 305-312.

Plotsky PM, Thrivikraman KV, Nemeroff CB, Caldji C, Sharma S, Meaney MJ (2005). Long-term consequences of neonatal rearing on central corticotropin-releasing factor systems in adult male rat offspring. Neuropsychopharmacology 30: 2192-2204.

Rainnie DG, Bergeron R, Sajdyk TJ, Patil M, Gehlert DR, Shekhar A (2004). Corticotrophin releasing factor-induced synaptic plasticity in the amygdala translates stress into emotional disorders. J Neurosci 24: 3471-3479.

Reul J (2002). Corticotropin-releasing factor receptors 1 and 2 in anxiety and depression. Curr Opin Pharmacol 2: 23-33.

Saavedra-Rodríguez L, Feig LA (2013). Chronic social instability induces anxiety and defective social interactions across generations. Biol Psychiatry 73: 44-53.

Schmidt HD, Duman RS (2010). Peripheral BDNF produces antidepressant-like effects in cellular and behavioral models. Neuropsychopharmacology 35: 2378-2391.

Schmidt MV, Scharf SH, Sterlemann V, Ganea K, Liebl C, Holsboer F et al (2010). High susceptibility to chronic social stress is associated with a depression-like phenotype. Psychoneuroendocrinology 35: 635-643.

Shepherd JK, Grewal SS, Fletcher A, Bill DJ, Dourish CT (1994). Behavioural and pharmacological characterisation of the elevated "zero-maze" as an animal model of anxiety. Psychopharmacology 116: $56-64$.

Spear LP (2000). The adolescent brain and age-related behavioral manifestations. Neurosci Biobehav Rev 24: 417-463.

Steimer T (2011). Animal models of anxiety disorders in rats and mice: some conceptual issues. Dialogues Clin Neurosci 13: 495-506.
Teicher MH, Andersen SL, Hostetter JC (1995). Evidence for dopamine receptor pruning between adolescence and adulthood in striatum but not nucleus accumbens. Brain Res Dev Brain Res 89: $167-172$.

Tsoory M, Cohen H, Richter-Levin G (2007). Juvenile stress induces a predisposition to either anxiety or depressive-like symptoms following stress in adulthood. Eur Neuropsychopharmacol 17: 245-256.

Treweek JB, Jaferi A, Colago EE, Zhou P, Pickel VM (2009). Electron microscopic localization of corticotropin-releasing factor (CRF) and CRF receptor in rat and mouse central nucleus of the amygdala. J Comp Neurol 512: 323-335.

Turner RJ, Lloyd DA (2004). Stress burden and the lifetime incidence of psychiatric disorder in young adults: racial and ethnic contrasts. Arch Gen Psychiatry 61: 481-488.

Wahlsten D. Mouse Behavioral Testing. Academic Press: San Diego, CA, 2010.

Walf AA, Paris JJ, Frye CA (2009). Chronic estradiol replacement to aged female rats reduces anxiety-like and depression-like behavior and enhances cognitive performance. Psychoneuroendocrinology 34: 909-916.

Walker DL, Davis M (1997). Double dissociation between the involvement of the bed nucleus of the stria terminalis and the central nucleus of the amygdala in startle increases produced by conditioned versus unconditioned fear. J Neurosci 17: 9375-9383.

Weiss IC, Franklin TB, Vizi S, Mansuy IM (2011). Inheritable effect of unpredictable maternal separation on behavioral responses in mice. Front Behav Neurosci 5: 3.

Whitaker-Azmitia PM (1991). Role of serotonin and other neurotransmitter receptors in brain development: basis for developmental pharmacology. Pharmacol Rev 43: 553-561.

Wiersma A, Baauw AD, Bohus B, Koolhaas JM (1995). Behavioural activation produced by $\mathrm{CRH}$ but not alpha-helical $\mathrm{CRH}$ (CRHreceptor antagonist) when microinfused into the central nucleus of the amygdala under stress-free conditions. Psychoneuroendocrinology 20: 423-432.

This work is licensed under a Creative Commons Attribution-NonCommercial-NoDerivs $\quad 4.0$ International License. The images or other third party material in this article are included in the article's Creative Commons license, unless indicated otherwise in the credit line; if the material is not included under the Creative Commons license, users will need to obtain permission from the license holder to reproduce the material. To view a copy of this license, visit http://creativecommons.org/licenses/by-nc-nd/4.0/

(C) The Author(s) 2017

Supplementary Information accompanies the paper on the Neuropsychopharmacology website (http://www.nature.com/npp) 